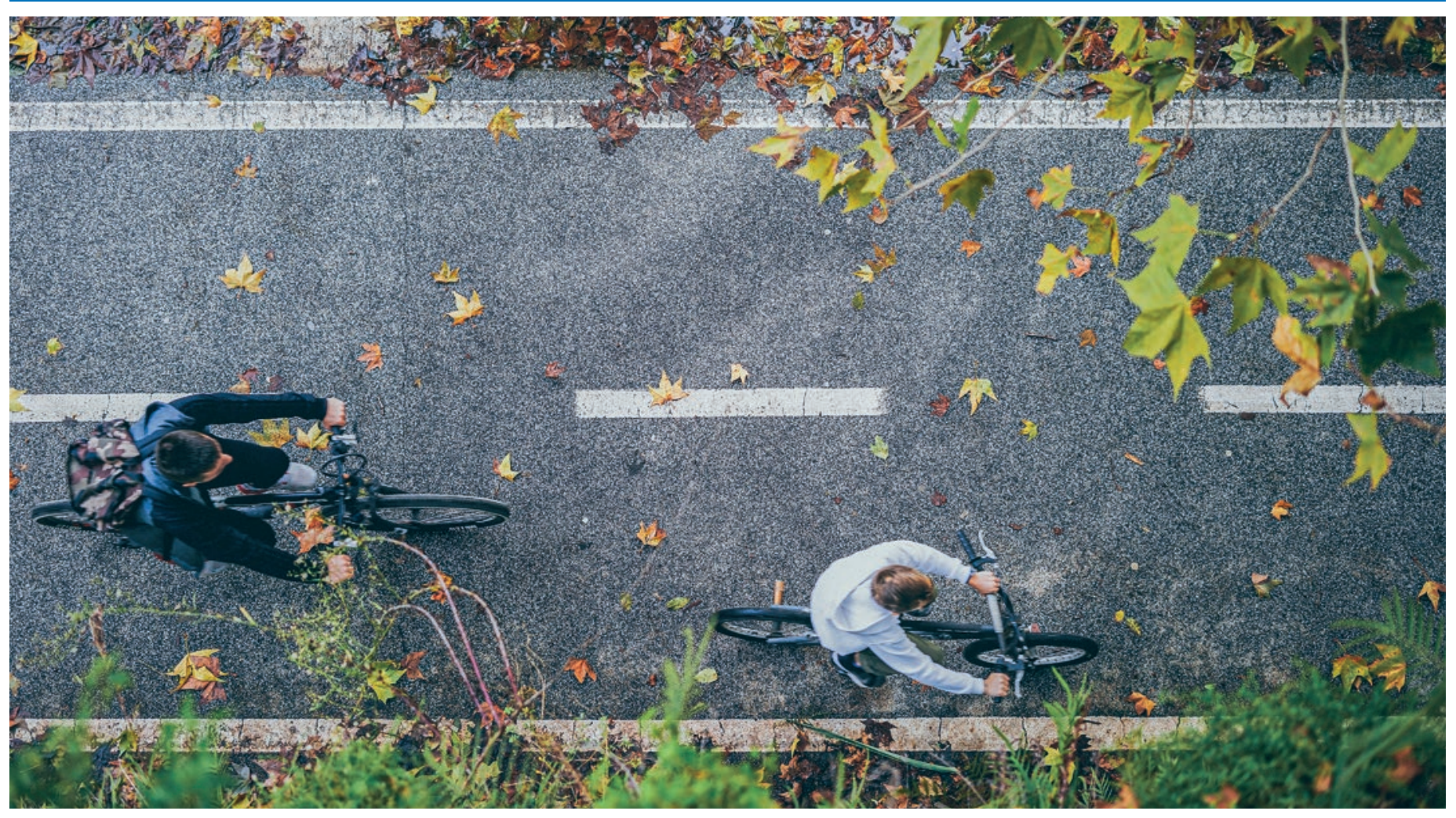

\title{
Wege entstehen dadurch, dass man sie geht
}

\section{Robin Riesera, Carlos Quintob ${ }^{\text {, Barbara Weilc }}$}

${ }^{a}$ Wissenschaftlicher Mitarbeiter Abteilung Public Health FMH; ${ }^{b}$ Mitglied des Zentralvorstandes der FMH, Verantwortlicher Depatement Public Health, Gesundheitsberufe und Heilmittel; ${ }^{c}$ Leiterin Abteilung Public Health, Gesundheitsberufe und Heilmittel

Der Klimawandel ist allgegenwärtig. Zu den Treibhausgasemissionen, welche diesen begünstigen, trägt der Gesundheitssektor wesentlich bei. Um der gemeinsamen Verantwortung in Sachen Klimawandel gerecht zu werden, hat die FMH die Strategie «Planetary Health - Strategie zu den Handlungsmöglichkeiten der Ärzteschaft in der Schweiz zum Klimawandel» verfasst. Diese wurde am 7. Oktober 2021 von der Ärztekammer verabschiedet.

Franz Kafka sagte: «Wege entstehen dadurch, dass man sie geht.» Über die letzten Jahrzehnte ging die Menschheit einen Weg, der nur in eine Richtung führt: Der Klimawandel ist angekommen - sowohl in der Schweiz als auch in den Köpfen der Bevölkerung. Unwetterkatastrophen des Sommers 2021 führten in der Schweiz und in weiten Teilen Europas zu immensen Schäden und Todesfällen. Diese häufiger auftretenden extremen Wetterlagen sind Teil der Klimaveränderung und damit auch der Veränderung der Ökosysteme in der Schweiz. Diese Veränderungen haben direkte und indirekte Auswirkungen auf die Gesundheit der gesamten Schweizer Bevölkerung (Abb. 1).
Hohe Temperaturen belasten den menschlichen Organismus. Hitze kann Erschöpfung und Hitzschlag auslösen sowie vorbestehende Erkrankungen wie Herz-Kreislauf-, Atemwegs-, Nieren- oder psychische Erkrankungen verschlimmern [1]. Weiter erhöhen Hitzeperioden das Risiko für Infektionen und Wundheilungsstörungen [2]. Hitzewellen und einzelne Hitzetage verursachten in der Schweiz sowohl eine $\mathrm{Zu}$ nahme von Notfall-Spitaleintritten [3] als auch eine Zunahme der allgemeinen Sterblichkeit $[4,5]$. Tropennächte sind ein zusätzliches Gesundheitsrisiko, da die nächtliche Erholung durch die fehlende Abkühlung beeinträchtigt wird [6]. 


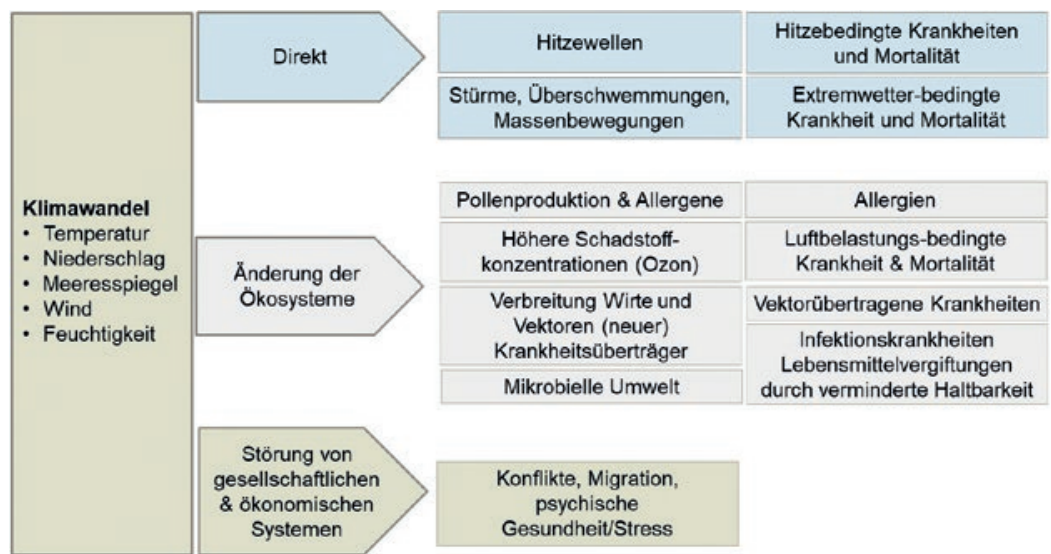

Abbildung 1: Direkte und indirekte Auswirkungen des Klimawandels auf die Gesundheit. Quelle: Swiss TPH, adaptiert von [8-10].

\section{Hitzewellen fördern Aggressionen}

Studien zufolge wirken sich hohe Temperaturen auch negativ auf die psychische Gesundheit aus. Untersuchungen in den USA und in Australien haben nachgewiesen, dass Hitzewellen Aggressionen fördern und die Kriminalitätsrate ansteigen lassen. Zusätzliche gesundheitliche Risiken im Zusammenhang mit sich verändernden Ökosystemen bestehen zudem durch die mögliche Ausbreitung von Krankheitsüberträgern, Krankheitserregern sowie allergenen Pflanzen. Ferner könnten die zukünftigen klimatischen Bedingungen eine Zunahme von über Wasser, Nahrungsmittel und Vektoren übertragenen Infektionskrankheiten begünstigen. Die Produktion und Haltbarkeit von Nahrungsmitteln hängt in vielfacher Weise mit den klimatischen Bedingungen zusammen, so dass Lebensmittelhygiene an Bedeutung gewinnt. Der Gesundheitssektor trägt wesentlich zu den nationalen Treibhausgasemissionen bei. International beträgt der Anteil des Gesundheitssektors an den Gesamtemissionen gemäss neustem Lancet Countdown-Bericht 4,6\% [1]. Besonders hoch ist der Anteil der $\mathrm{CO}_{2}$-Emissionen des nationalen Gesundheitssystems an den Gesamtemissionen mit zwischen 8 und 10\% [6, 7] beispielsweise in den USA. In Grossbritannien betrug 2017 der entsprechende Anteil 4 bis 5\%. Aufgrund der Bestrebungen des National Health Service (NHS) [8], das weltweit erste Netto-Null-Gesundheitssystem zu werden, sinkt er seither [1]. Die Zahlen für die Schweiz unterscheiden sich je nach Studie und Berechnungsmethode. Ein internationaler Vergleich des $\mathrm{CO}_{2}$-Fussabdrucks des Gesundheitssektors von Pichler et al. [9] geht für die Schweiz im Jahr 2014 von einem Anteil von 5,9\% aus. Die internationale Organisation Health Care Without Harm hat diesen Wert für 2019 auf 6,7\% geschätzt [10]. Zahlen von nationalen Studien gibt es bisher keine.
Die Deutlichkeit der Klimaveränderungen und der einhergehenden Konsequenzen ist spätestens seit der Veröffentlichung des Sixth Assessment Report des Intergovernmental Panels on Climate Change (IPCC) nicht mehr von der Hand zu weisen [11]. Die Eindämmung des Klimawandels - und damit der Schutz unserer Gesundheit und der Gesundheit der kommenden Generationen - sollte unser Handeln bereits heute leiten.

\section{Wir alle tragen Verantwortung}

Vielerorts besteht die Gefahr, dass die in der Vergangenheit erzielten Fortschritte bei der globalen Gesundheit durch die globale Erwärmung beeinträchtigt werden. Die Hochwasser, Starkregen, Waldbrände und Dürren des Sommers 2021 und der im August 2021 veröffentlichte Sixth Assessment Report des IPCC [11] haben eindrücklich gezeigt, dass wir alle die Verantwortung tragen, unseren Teil zum Schutz des Klimas und der öffentlichen Gesundheit beizutragen. Der NHS hat sich bereits vor 10 Jahren für den Weg des Klimaschutzes entschieden [8]. Seine Anstrengungen fokussieren sich auf Organisationen und Individuen, beispielsweise auch auf die Hausärztinnen und Hausärzte.

\section{Der Klimawandel ist angekommen - in der} Schweiz und in den Köpfen der Bevölkerung.

Das Green Impact for Health Toolkit des Royal College of General Practicioners (RCGP) bietet Möglichkeiten, Nachhaltigkeit über verschiedene Themen in die tägliche Praxisarbeit zu integrieren. Es beschreibt an den Sustainable Development Goals (SDGs) der UNO orientierte Massnahmen, die nach Bedarf und Möglichkeit Schritt für Schritt umgesetzt werden können. Beispielsweise im Bereich Abfall und Recycling: Wird der in der Praxis entstehende Abfall getrennt, Druckerpapier aus Recyclingpapier genutzt und Altpapier recycelt, erreicht die Praxis das bronze level Abfall und Recycling. Weitere Level (silver, gold und carbon) beinhalten die Reduktion von Einwegplastik und persönlichem Schutzmaterial, Handhabung ungenutzter Medikamente, Lagerhaltung und Bestellwesen, Auswahl der Büromaterialien etc.

Werden Massnahmen über alle 13 Bereiche umgesetzt, besteht durch ein Auditverfahren sogar die Möglichkeit, die eigene Praxis auszeichnen zu lassen oder den Green Impact award zu erhalten. Der stufenweise Aufbau der Massnahmen und der einfache Zugang über eine Online-Plattform machen das Green Impact for Health Toolkit des RCGP zu einer verhältnismässigen und realistischen Massnahme, um die Erreichung der SDGs und den Schutz des Klimas voranzutreiben. 


\section{Massnahmen in der Schweiz}

Bereits heute finden sich in der ganzen Schweiz innovative Projekte, die sich der Umsetzung der SDGs annehmen. Das Kantonsspital Luzern setzte im Zeitraum von 2017 bis 2020 ein Projekt zur Reduktion der Lebensmittelverschwendung (Foodwaste) um. Durch wenige Massnahmen konnte eine Reduktion von 35\% erreicht werden. Dies entspricht ungefähr 150000 Tonnen weniger Lebensmittelabfällen.

Das 2000-Watt-Areal setzt sich zum Ziel, Siedlungsgebiete zu schaffen, die es den Bewohnern ermöglichen, energieeffizient und nachhaltig zu leben. In die Planung werden unter anderem Aspekte von Mobilität, Grünflächen, Nutzung, Baumaterialien und Energie miteinbezogen. Das Projekt Green Hospital evaluiert die Ressourceneffizienz der Schweizer Spitäler, um zu ermitteln, welche Prozesse optimiert werden können und wo ökonomisches und ökologisches Sparpotenzial besteht.

\section{Studien zufolge wirken sich hohe Temperaturen} auch negativ auf die psychische Gesundheit aus.

Der Kanton Bern hat am 26. September 2021 als zweiter Kanton der Schweiz entschieden, den Klimaschutz in die Verfassung aufzunehmen. Der Kanton Bern will damit bis 2050 klimaneutral sein, er will sich mit den Gemeinden aktiv für eine Begrenzung der Klimaveränderung und deren nachteiliger Auswirkungen einsetzen. Diese Projekte schlagen den Weg der nachhaltigen Nutzung unserer Ressourcen ein und zeigen, dass es möglich ist, gleichzeitig wettbewerbsfähig und klimafreundlich handeln zu können.

\section{Manifest für eine gesunde Zukunft}

Die Delegiertenversammlung der FMH hat an ihrer Sitzung vom 3. September 2020 den Klimawandel als substantielle Bedrohung für die regionale und globale Gesundheit anerkannt und im Namen der Schweizer Ärzteschaft das "Manifest für eine gesunde Zukunft» unterschrieben. Um der gemeinsamen Verantwortung in Sachen Klimawandel gerecht zu werden, hat die FMH die Strategie «Planetary Health - Strategie zu den Handlungsmöglichkeiten der Ärzteschaft in der Schweiz zum Klimawandel» verfasst, die am 7. Oktober 2021 von der Ärztekammer der FMH verabschiedet wurde.

Die Strategie umfasst Ziele in vier verschiedenen Handlungsbereichen: Information, Reduktion, Adaptation und Vorbildrolle, und verfolgt die Vision eines nachhaltig gesundheitsfördernden und klimaresilienten Schweizer Gesundheitswesens. Die Schweizer Ärzteschaft unterstützt eine Verstärkung der Mass- nahmen gegen den Klimawandel und hilft mit, die Zielsetzung eines umweltfreundlichen Schweizer Gesundheitswesens mit verhältnismässigen, finanziell tragbaren Massnahmen zu erreichen.

Zur Erreichung des auch von der Schweiz ratifizierten Übereinkommens von Paris [12] und der Umsetzung der SDGs braucht es jedoch mehr als die Anstrengungen der Ärztinnen und Ärzte, Spitäler oder Kantone und Gemeinden. Alle sollten gemeinsam die Bewältigung der Klimakrise angehen. Nur durch gut koordinierte Massnahmen können wir gemeinsam einen Beitrag leisten, das Klima und dadurch die Gesundheit aller in der Schweiz lebenden Personen zu schützen und den Weg für eine nachhaltige und gesundheitsfördernde Zukunft zu ebnen.

\section{Bildnachweis \\ Ümit Ylldırım / Unsplash}

\section{Literatur}

1 Watts N, Amann M, Arnell N, et al. The 2020 report of The Lancet Countdown on health and climate change: responding to converging crises. The Lancet. Published online 2020.

2 Aghdassi SJS, Schwab F, Hoffmann P, Gastmeier P. The Association of Climatic Factors with Rates of Surgical Site Infections. Dtsch Aerzteblatt Int. 2019;116.

3 Ragettli MS, Vicedo-Cabrera AM, Flückiger B, Röösli M. Impact of the warm summer 2015 on emergency hospital admissions in Switzerland. Environ Health. 2019;18(66):1-10.

4 Ragettli MS, Röösli M. Gesundheitliche Auswirkungen von Hitze in der Schweiz und die Bedeutung von Präventionsmassnahmen. Hitzebedingte Todesfälle im Hitzesommer 2019 - und ein Vergleich mit den Hitzesommern 2003, 2015 und 2018. Schweizerisches Tropen- und Public Health-Institut (Swiss TPH); 2020. https://www.nccs.admin.ch/dam/nccs/de/dokumente/website/ sektoren/gesundheit/bericht-gesundheitliche-auswirkungen von-hitze-in-der-schweiz-und-die-bedeutung-von-praeventionsmassnahmen-korrigier.pdf.download.pdf/SwissTPH_2020_ Gesundheitliche\%20Auswirkungen\%20von\%20 Hitze 2019 Vergleich\%202003-2015-2018 def corrected.pdf

5 Vicedo-Cabrera AM, Ragettli MS, Schindler C, Röösli M. Excess mortality during the warm summer of 2015 in Switzerland. Swiss Med Wkly. 2016;146:w14379.

6 Ragettli MS, Vicedo-Cabrera AM, Schindler C, Röösli M. Exploring the association between heat and mortality in Switzerland between 1995 and 2013. Environ Res. 2017;158C:703-9.

7 BAFU. Klimabedingte Risiken und Chancen. Eine schweizweite Synthese. Bundesamt für Umwelt (BAFU). 2017:148.

8 NHS England NI. Delivering a 'Net Zero' National Health Service. Published online 2020. Accessed July 14, 2021. https://www.england.nhs.uk/greenernhs/publication/delivering-a-net-zeronational-health-service/

9 Pichler PP, Jaccard IS, Weisz U, Weisz H. International comparison of health care carbon footprints. Environ Res Lett. 2019;14(6): 064004.

10 Health Care Without Harm US \& Canada. Health care's climate footprint. How the health sector contributes to the global climate crisis and opportunities for action. Published 2019. Accessed January 31, 2021. https://noharm-uscanada.org/ClimateFootprintReport

11 IPCC. Climate Change 2021: The Physical Science Basis. Contribution of Working Group I to the Sixth Assessment Report of the Intergovernmental Panel on Climate Change [Masson-Delmotte V, Zhai P, Pirani A, Connors SL, Péan C, Berger S, Caud N, Chen Y, Goldfarb L, Gomis MI, Huang M, Leitzell K, Lonnoy E, Matthews JBR, Maycock TK, Waterfield T, Yelekçi O, Yu R and Zhou B (Eds.)] Cambridge University Press. In Press; 2021.

12 BAFU (Hrsg.) 2018: Klimapolitik der Schweiz. Umsetzung des Übereinkommens von Paris.

Bundesamt für Umwelt, Bern. Umwelt-Info Nr. 1803: 28 S. 